\title{
On the Micrometeorology of the Southern Great Plains. 2: Turbulence Statistics
}

\author{
Bruce B. Hicks - W. R. Pendergrass III • C. A. Vogel • \\ R. N. Keener Jr. • S. M. Leyton
}

Received: 6 February 2014 / Accepted: 29 October 2014 / Published online: 21 November 2014

(C) The Author(s) 2014. This article is published with open access at Springerlink.com

\begin{abstract}
Fast-response micrometeorological data obtained from an instrumented 32-m tower at an arid site near Ocotillo, Texas are used to examine the daily time evolution of the lower atmosphere. Correlation coefficients between turbulence properties (fast response wind-speed components and temperature) confirm that over this sparsely vegetated site the effects of convection are observed soon after sunrise, well ahead of the morning transition from stable to unstable stratification. Details of this kind are obscured when results are considered as functions of conventional stability parameters, since such standard analytical methods combine features of the morning and evening transitions into a single presentation. Partial correlation coefficients and semi-partials indicate that the local turbulent kinetic energy is mainly associated with local fluxes of heat and momentum near neutral and in most stable conditions, but decreases substantially during the times of strongest instability (possibly reflecting the scatter introduced by sampling infrequent convective episodes using a single tower). For many of the variables considered here, the standard deviations are about the same as the linear averages, indicating that the distributions are close to log-normal. The present data indicate that if the intent is to address some specific situation then $\pm 10 \%$ error bounds on turbulence quantities (e.g. fluxes) correspond to averaging over a distance scale of the order of $10 \mathrm{~km}$ and a time scale of about $3 \mathrm{~h}$. As the distance and time scales become smaller, the uncertainties due to factors external to the local surface increase.
\end{abstract}

\author{
B. B. Hicks $(\varangle)$ \\ MetCorps, P. O. Box 1510, Norris, TN 37828, USA \\ e-mail: hicks.metcorps@gmail.com \\ W. R. Pendergrass III - C. A. Vogel \\ NOAA/ARL/ATDD, P. O. Box 2456, Oak Ridge, TN 37831, USA \\ C. A. Vogel \\ Oak Ridge Associated Universities, P. O. Box 117, Oak Ridge, TN 37831, USA \\ R. N. Keener Jr. · S. M. Leyton \\ Duke Energy, 26 South Church Street, Charlotte, NC 28202, USA
}


Keywords Free convection - Surface boundary layer - Turbulence kinetic energy ·

Turbulence statistics

\section{Introduction}

In Part I, Hicks et al. (2014) present the results of a micrometeorological flux and gradient study conducted near Ocotillo in western Texas $\left(32.12050^{\circ} \mathrm{N} ; 101.37555^{\circ} \mathrm{W}\right)$. Data were obtained over a 6-month period, using sonic anemometers and ventilated platinum resistance thermometers at seven heights on a 32-m tower. The instruments were mounted on horizontal arms extending beyond the tower to a distance at which interference due to the presence of the tower was minimized. Figure 1 shows the experimental set-up; the site is in a poorly vegetated, arid area, fairly typical of the Southern Great Plains but lacking the surface homogeneity of many "outdoor laboratory" micrometeorological studies. The area is well known for its high winds, mainly from the south, and as a consequence has been selected for wind "farming." To avoid the influence of the turbines, the tower of major interest here was installed to the south (and upwind) of the turbine field. In the analysis to follow, winds from the sector

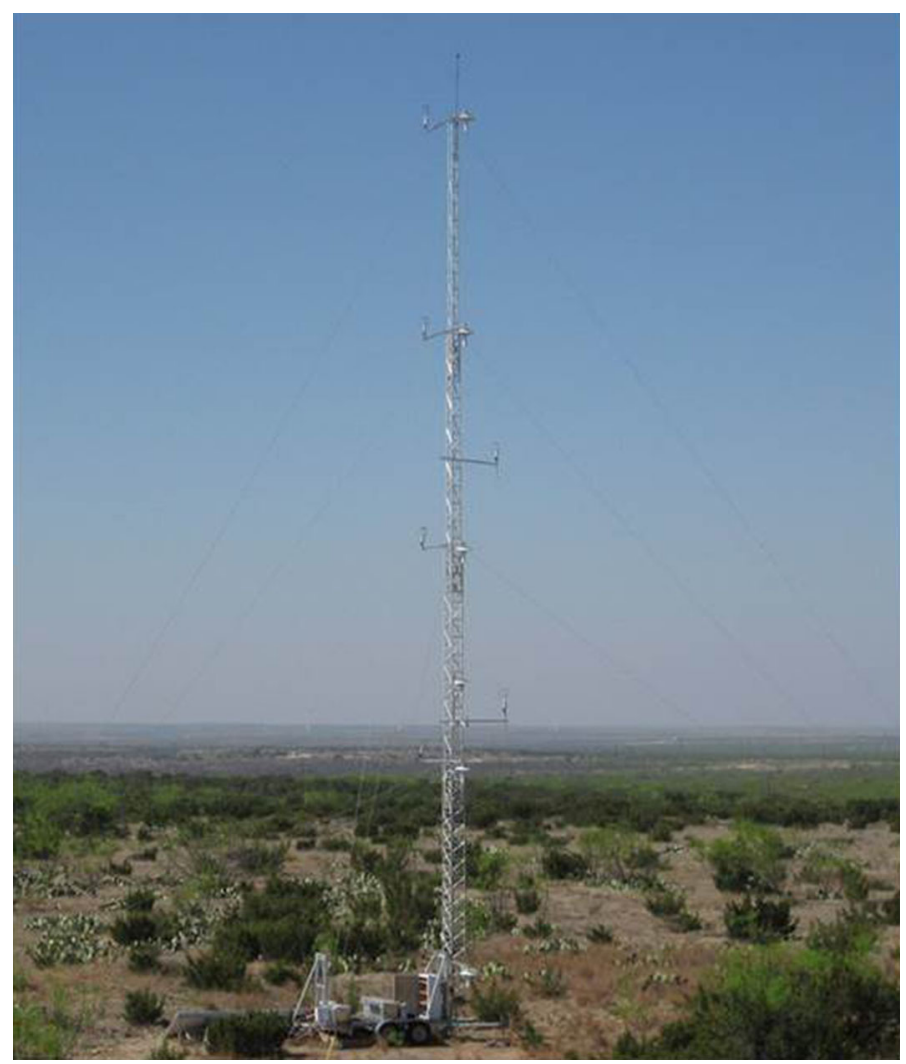

Fig. 1 A view of the 32-m tower located near Ocotillo, Texas $\left(32.12050^{\circ} \mathrm{N} ; 101.37555^{\circ} \mathrm{W}\right)$, showing the upwind area for the dominant southerly winds. Note that the arms carrying instruments are not all pointing in the same direction, but are arranged to capture the prevailing mean wind 
possibly affected by the wind turbines, $280^{\circ}$ through north to $080^{\circ}$, have been excluded. The photograph of Fig. 1 is taken looking to the south, into the prevailing wind direction. The arms carrying the instrumentation are arranged accordingly, while also being organized to minimize any potential interference between sensors at adjacent heights.

Surrounding vegetation is mainly mesquite and buffalo grass growing to a height as much as $2 \mathrm{~m}$. The zero-plane displacement $(d)$ was previously estimated to have been about $0.8 \mathrm{~m}$, and the aerodynamic roughness length about $0.18 \mathrm{~m}$. Sonic anemometer and resistance thermometer data are available for seven heights (z) 3.0, 8.5, 10.0, 14.7, 17.2, 21.0, and $27.4 \mathrm{~m}$. The data used here were generated using 15-min runs around the clock without exception. It is acknowledged that this represents a departure from contemporary micrometeorological practice, in which 30-min or 60-min runs have become standard. The choice of a shorter run length was made to provide greater time resolution, of special interest when considering the dawn and dusk transition periods. The consequences of using a short sampling period are discussed later.

One particular purpose of the Ocotillo study was to test the applicability of legacy fluxgradient relations now that new instrumentation enables a closer look at the processes involved. Despite overall agreement with the commonly accepted flux-gradient relationships, the analysis reported in Hicks et al. (2014) illustrates the uncertainties arising when these formulations are used to describe individual 15-min runs. For the individual runs, classification by bulk Richardson number indicates little correlation between 15-min friction velocities $\left(u_{*}\right)$ and vertical wind gradients in stable conditions, although wind and temperature gradients ( $d u / d z$ and $d \theta / d z$, respectively) are then closely related. This close association is attributed to the heat exchange being a consequence of the prevailing temperature gradient and turbulence regime, with the former resulting from radiative cooling of the surface and the latter being velocity-driven. In unstable stratification, the data show evidence of free convection, over the stability range $Z / L<-0.03$ (where $Z=z-d$ ), in line with estimates obtained in some of the earliest micrometeorological studies (in the 1960s and earlier, q.v. Priestley 1955).

The present intent is to explore the Ocotillo data further, through examination of characteristic turbulence statistics and especially of, (a) the correlations among the primary quantities, (b) the variability of individual cases around the averages that are most often considered, and (c) the extent to which turbulent kinetic energy (TKE) is determined by local fluxes. The present analysis is intended to address issues related to two distinct applications-mesoscale modelling and plume dispersion. Models addressing the first of these two issues characteristically make use of non-dimensionalized flux-gradient relationships that assume the dominant role of surface fluxes. Dispersion models focus on wind-speed and wind-direction variability, described statistically as functions of height and stability. The Ocotillo data allow tests of these and other common assumptions.

Data have been subjected to coordinate rotation to align the results with the mean horizontal wind vector and to drive the vertical component to zero. Thus, in the momentum datasets considered here the average vertical and transverse velocity components are both zero. The temperature $(T)$ covariance data are not rotated, since buoyancy is controlled by gravity, the instruments were carefully aligned according to gravity, and angles of correction were always less than two degrees. It is acknowledged that the values of $\overline{w^{\prime} T^{\prime}}$ used here are likely to be underestimates; the present study made use of the same sonic anemometers as reported by Kochendorfer et al. (2012) as having a possible $10 \%$ underestimation in $\overline{w^{\prime} T^{\prime}}$ 

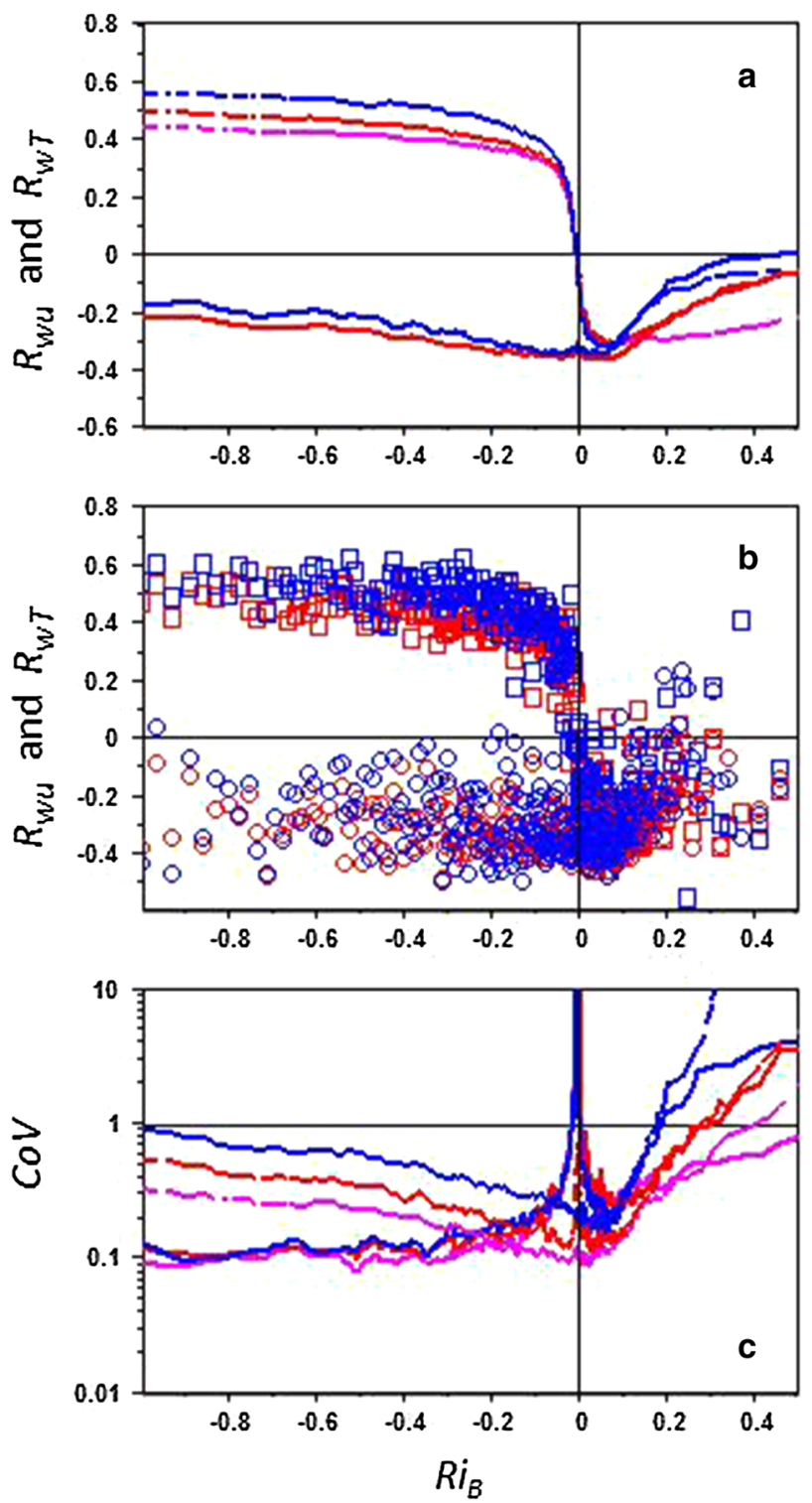

Fig. 2 The variation with bulk Richardson number $\left(R i_{B}\right)$ of the correlation coefficients $R_{w u}$ and $R_{w T}(\mathbf{a})$ and (b) and of the corresponding coefficients of variation (c) for data from $3.0 \mathrm{~m}\left(R_{w u}\right.$ only: magenta), $8.5 \mathrm{~m}$ (red), and $21.0 \mathrm{~m}$ (blue). The uppermost panel shows the trends of average values constructed after ordering according to $R i_{B}$. The middle panel illustrates the scatter of the individual data points: every fiftieth point is plotted. The bottom panel shows the variations in the coefficient of variation ( $\mathrm{CoV}$, the ratio of the standard deviation to the average), lighter dashed lines refer to $R_{w u}$ data, solid lines to $R_{w T}$

\section{Correlations}

To test the stability dependence of turbulence statistics normally addressed in terms of $Z / L$, Fig. 2a shows how the correlation coefficients between the longitudinal $(u)$ and vertical $(w)$ 
wind components $\left(R_{w u}\right)$ and between the temperature $(T)$ and the vertical wind component $\left(R_{w T}\right)$ vary according to $R i_{B}$, the Richardson number based on the 8.5-m and 21.0-m data. The curves are drawn to connect averages of groups of fifty 15-min runs, arranged sequentially after ordering by $R i_{B}$. For the $R_{w T}$ case, data from three levels are illustrated- $3.0 \mathrm{~m}$ (magenta), $8.5 \mathrm{~m}$ (red) and $21.0 \mathrm{~m}$ (blue). For the $R_{w u}$ case, the $3.0-\mathrm{m}$ results are not shown. (Close inspection of the data reveals that the velocity data at $3.0 \mathrm{~m}$ were occasionally too close to the tops of surrounding plants. This does not appear to be the case for the temperature data, raising questions about the effective height of measurement and the relevant zero plane that will be addressed later.) For the data illustrated, both $R_{w u}$ and $R_{w T}$ appear well behaved, with results for the other heights falling in order with the traces shown. Several well-known features of the surface boundary layer can be recognized: (a) as instability increases, $R_{w T}$ approaches a value between 0.5 and 0.6 , depending on height, whereas the magnitude of $R_{w u}$ slowly diminishes; (b) in stable conditions, the correlation coefficients tend towards zero least rapidly for the lowest heights; (c) the transition through neutral appears to be rapid and mostly confined to the range $-0.1<R i_{B}<0.1$. Here, the planetary boundary layer (PBL) is visualized as the lowest part of the atmosphere that responds to both the diurnal cycle and spatial changes in the surface. The surface boundary layer (SBL) is that part of the PBL within which fluxes are closely constant with height, viz the "constant-flux layer."

Figure 2a shows consistent behaviours affecting average values, while Fig. $2 b$ illustrates the scatter obscured by the averaging of Fig. 2a. To avoid presenting an uninterpretable cloud of data points, every fiftieth value is plotted, after ordering by $R i_{B}$. In Fig. $2 \mathrm{~b}$, red indicates 8.5-m data, and blue indicates 21.0-m data; squares show $R_{w u}$ and circles show $R_{w T}$. It is clear that scatter is a dominant factor, with the most ordered behaviour occurring for the temperature correlation in unstable conditions.

Figure $2 \mathrm{c}$ summarizes the statistical variability in terms of the ratio of the standard deviation $\sigma(x)$ to the modulus of the mean value $|\bar{x}|$. This ratio is the basis for a number of statistical tests, and is classically referred to as the coefficient of variation $(\operatorname{CoV})$. For $|\bar{x}| \gg 0$, a low CoV value indicates a tightly-confined statistical distribution of the relevant variable, while a high value (approaching or exceeding unity) indicates a distribution largely determined by variables that are disordered, bordering on log-normal. In this context, Fig. 2c has no surprises: the sensible heat correlation is well behaved through all instabilities except for very near-neutral, and for a small portion of the stable regime. However, as stability increases CoV quickly approaches unity, indicating that the underlying quantities ( $w$ and $T$ in this case) become poorly related. This was evident earlier, in Fig. $2 \mathrm{a}$.

The conventional view of the transition between unstable and stable stratification is supported by the data of Fig. 2. On the average, the $R_{w T}$ transitions appear to extend over a range of values of $R i_{B}$ from about -0.2 to 0.1 . Over this range, there is little variation evident in the values of $R_{w u}$. The earlier analysis of these data, focusing on how the data compare with commonly accepted descriptions of the flux-gradient relations (Hicks et al. 2014), showed that there is benefit in considering the transition periods more carefully. Figure 3 shows how the onset of daylight affects the correlations. The depictions of the data in Fig. 3 follow those of Fig. 2, except that in the case of Fig. 3 the data have first been ordered according to the time relative to local sunrise. Sunrise times (and sunset times) were derived using the NOAA Earth System Research Laboratory solar calculator (http://www.esrl.noaa.gov/gmd/ $\mathrm{grad} / \mathrm{solcalc} /$ ). Data for each day were then arranged by time, (a) relative to this sunrise times, and alternatively (b) relative to the sunset time for the same day.

The $R_{w T}$ correlation coefficients plotted in Fig. 3a show a rapid change at about the time of sunrise, from the comparatively stationary nighttime value to a new, nearly uniform, regime after about $2 \mathrm{~h}$ and extending to a few hours before sunset (occurring about $12 \mathrm{~h}$ 

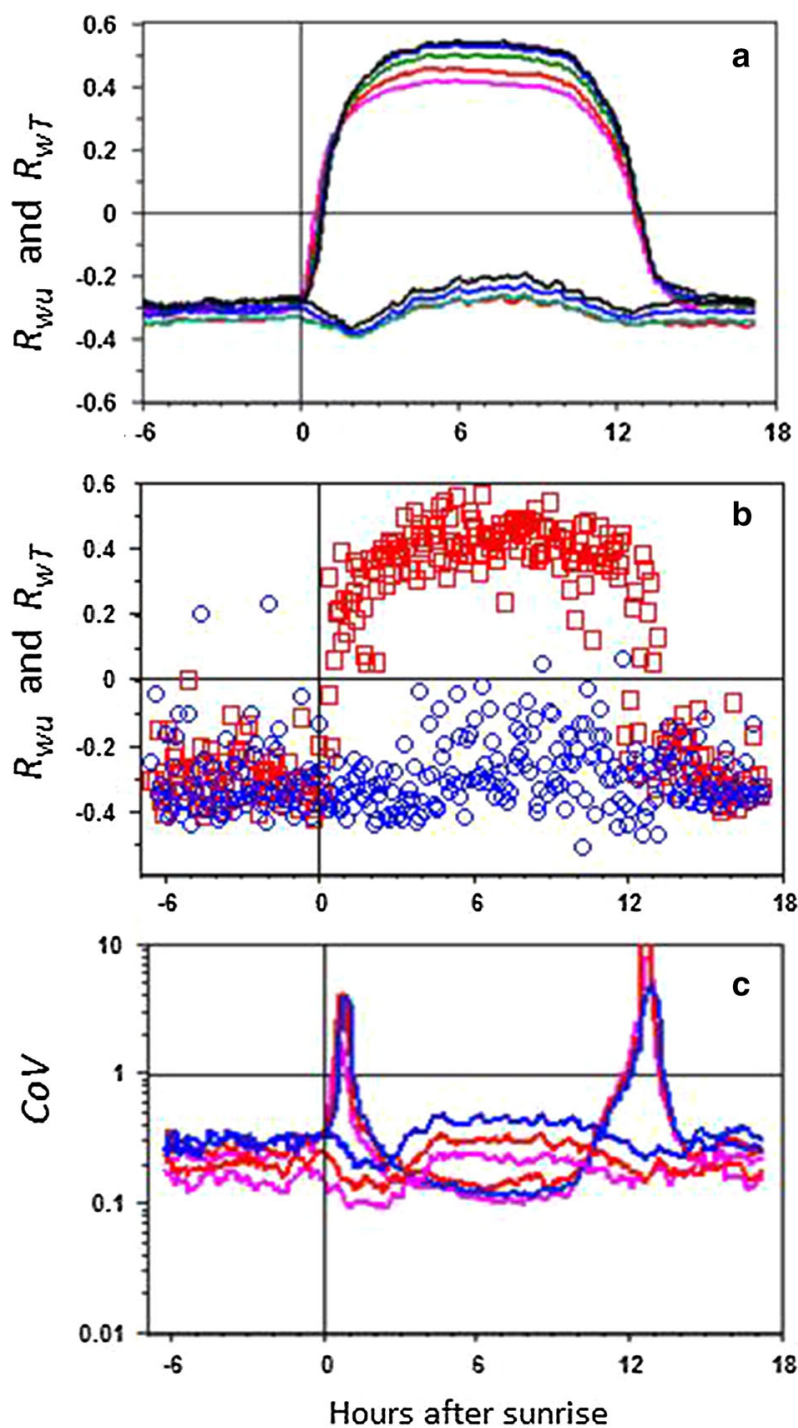

Fig. 3 As in Fig. 2, but plotting against the time of day, referenced to sunrise. In Fig. 3b, data are all from $14.7 \mathrm{~m}$ : red for $R_{w T}$, blue for $R_{w u}$. Otherwise, data relate to heights of $3.0 \mathrm{~m}$ (magenta), $8.5 \mathrm{~m}(\mathrm{red}), 14.7 \mathrm{~m}$ (green), $21.0 \mathrm{~m}$ (blue) and $27.4 \mathrm{~m}$ (black)

after sunrise, on the average). There is no evidence of a change in the characteristics of the free convection regime through this plateau region ( $2-10 \mathrm{~h}$ after sunrise). The average $R_{w T}$ values consistently increase with height, but not so $R_{w u}$.

Data from only one height $(14.7 \mathrm{~m})$ are presented in Fig. 3b, these being sufficient to show the scatter of the individual measurements. This scatter appears to be fairly constant with time, resulting in dominating peaks in the coefficient of variation for $R_{w T}$ (Fig. 3c) near dawn and dusk when the sensible heat flux is smallest. These peaks also serve to emphasize an over-riding reality of the present analysis - there is no allowance for the presence of clouds or for the delay in the transitions occasioned by them. The result is that the sunrise and sunset 

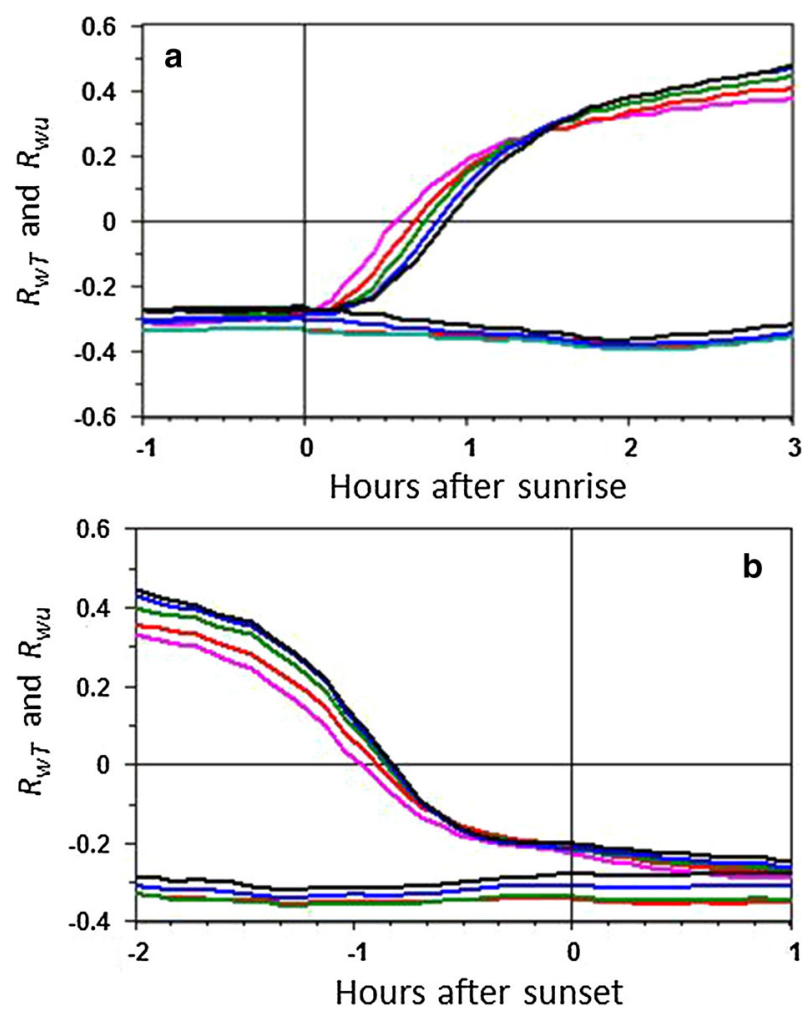

Fig. 4 Details of the transitions through sunrise (a) and sunset (b) of the average correlation coefficients $R_{w u}$ and $R_{w T}$ for five of the heights of measurement: $3.0 \mathrm{~m}$ (magenta), $8.5 \mathrm{~m}$ (red), $14.7 \mathrm{~m}$ (green), $21.0 \mathrm{~m}$ (blue), and $27.4 \mathrm{~m}$ (black)

transitions of the present focus are doubtlessly smoothed and prolonged by the occasional presence of clouds, with unknown influence on the breadth of the $C o V$ peaks summarized for $R_{w T}$ in Fig. 3c.

Details of the sunrise and sunset transitions are shown in Fig. 4, where data from several levels of measurements are plotted for $R_{w T}$, but for fewer levels for $R_{w u}$. Before dawn, all levels display the same average value of $R_{w T}$. At sunrise, Fig. 4 a shows that the lowest level responds first, with the other levels responding in sequence until the 27.4-m level crosses into instability (at $R_{w T}=0$ ) about $15 \mathrm{~min}$ after the 3-m level. In this early stage of convection, the $R_{w T}$ correlation coefficient decreases with height. However, at about $1.5 \mathrm{~h}$ after sunrise the variation with height changes sign: $R_{w T}$ then increases with height, as evident in Fig. $2 \mathrm{a}$. Note that this crossover refers to a behaviour among data ensembles, and involves data from all of the heights reported. It is a striking feature of Fig. 4, with a clear physical explanation not yet apparent but possibly corresponding to the "breakthrough" when deep convection starts to dominate. An alternative explanation associated with the present reliance on 15-min data and the resulting neglect of contributions of very low frequency eddies has been tested using a subsequent record of 60-min average data, which provides supporting evidence of the same crossover but with less clarity.

As sunset approaches (as in Fig. 4b), the average values of $R_{w T}$ decrease and data from the different levels converge. As expected, there is no abrupt change that corresponds to the 


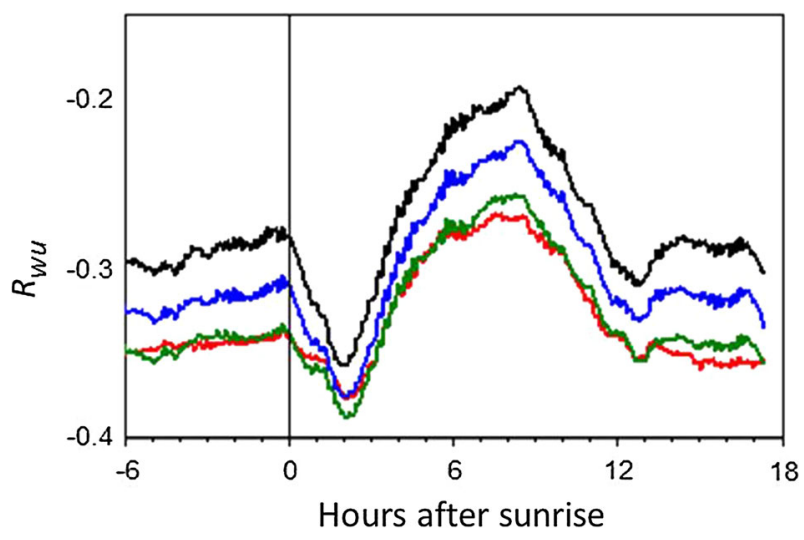

Fig. 5 An expanded view of the changes with time of day after sunrise of the momentum correlation coefficient $R_{w u}$. Red, green, blue and black refer to data from heights of 8.5-m, 14.7-m, 21.0-m, and 27.4-m levels respectively

time of actual sunset, since convection certainly continues as permitted by the heat storage in the soil and the overlying canopy. An obvious repercussion of this last observation is that the overall situation must necessarily be site specific, with biomass a key consideration.

Sorting according to time after sunrise yields a result for $R_{w u}$ that differs from that arising from the earlier grouping according to $R i_{B}$ : there is indeed a height dependence of $R_{w u}$ evident in Fig. $4 \mathrm{a}$, with the highest value of $\left|R_{w u}\right|$ being nearest the surface. This is illustrated in more detail in Fig. 5, where the morning transition is depicted with expanded scales. The curves plotted in Fig. 5 emphasize the consistent variation peaking about $2 \mathrm{~h}$ after dawn, when the value of $R_{w u}$ attains a minimum (on the average). Since the values of $R_{w u}$ are necessarily negative, this implies that the transfer of momentum is more effective at this time, diminishing as time progresses and as convection starts to dominate.

\section{Attribution of Turbulent Kinetic Energy}

Consider the TKE, $e=\left(\sigma_{u}^{2}+\sigma_{v}^{2}+\sigma_{w}^{2}\right) / 2$, and its association with local fluxes. The analysis above uses results obtained after ordering the data into sequential 50-run groups, according to stability or time of day. This same grouping procedure permits examination of the dependence of the local TKE on the local heat and momentum fluxes. Within every fifty-member group, correlations have been computed among three variables: $e, u_{*}$ and $\overline{w^{\prime} T^{\prime}}$, considered as variables 1, 2 and 3 respectively. From the three correlation coefficients $\left(R_{12}, R_{23}\right.$, and $\left.R_{31}\right)$, partial correlation coefficients have been evaluated: $R_{12.3}$ and $R_{13.2}$. These can be interpreted as, e.g., the correlation between variables 1 and 2 after the effect of variable 3 is accounted for. That is, the correlation of $e$ with $u_{*}$ if the influence of $\overline{w^{\prime} T^{\prime}}$ is removed. Partial correlation coefficients are plotted in Fig. 6, with a sign convention whereby an increase corresponds to an increase in the production of TKE. It is seen that for a wide spread of instabilities the role of $u_{*}$ is minimal.

Figure 7 extends this analysis by showing estimates of the local TKE that can be attributed to the combined effects of local heat and momentum fluxes. This analysis makes use of the semi-partial correlation methodology outlined by de Heus (2012), which results in the evaluation of the proportion of the total variance in $e$ that is attributable to the joint effects of $u_{*}$ and $\overline{w^{\prime} T^{\prime}}$ as 

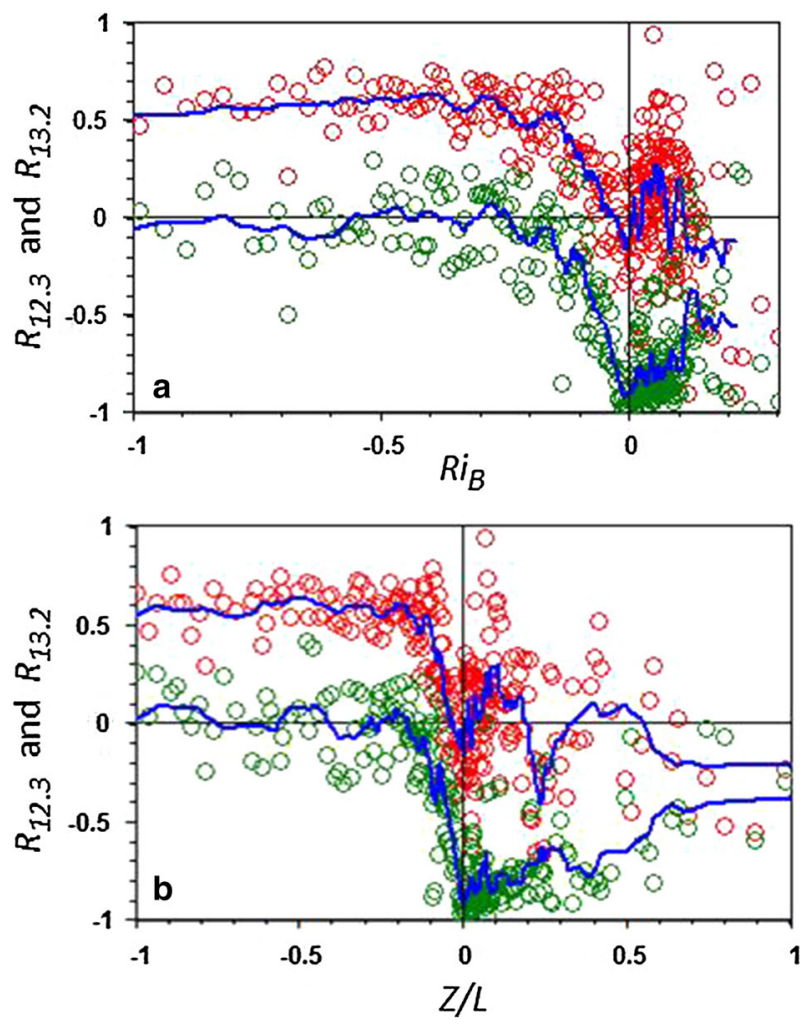

Fig. 6 Partial correlation coefficients derived for the 14.7-m level, showing (a) the correlation between TKE and $\overline{w^{\prime} T^{\prime}}$ if $u_{*}$ is held constant (red) and the correlation of TKE and $u_{*}$ if $\overline{w^{\prime} T^{\prime}}$ is held constant (green). Every fiftieth point is plotted, after ordering by $R_{B}$ (a) and by $Z / L(\mathbf{b})$. The blue lines are drawn through the average values of the 50-point groupings

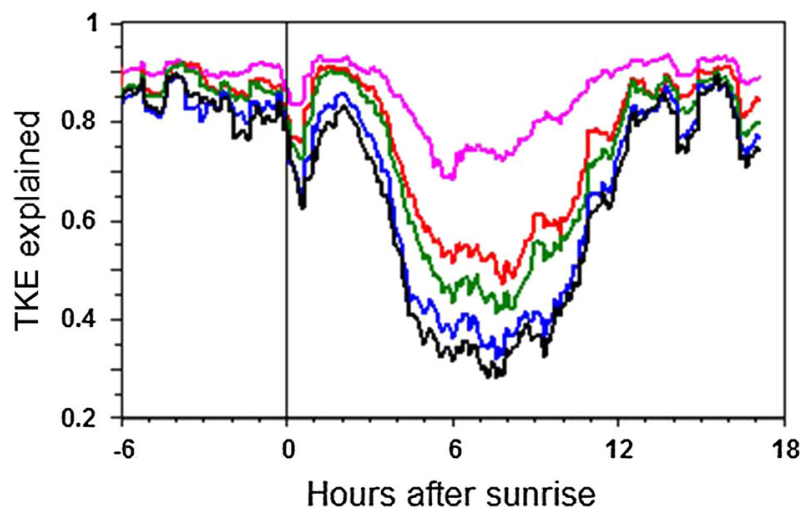

Fig. 7 The proportion of the observed TKE that can be explained in terms of local contributions, represented

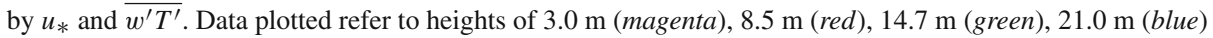
and $27.4 \mathrm{~m}$ (black). Results for levels of $10 \mathrm{~m}$ and $17.2 \mathrm{~m}$ lie in the expected sequence 


$$
R_{1.23}^{2}=R_{1.23}^{2}\left(1-R_{13}^{2}\right)+R_{13}^{2}=R_{13.2}^{2}\left(1-R_{12}^{2}\right)+R_{12}^{2} .
$$

In Fig. 7, results are shown for a selection of the heights of measurement, ordered by time of day; for most of the day, local measurements account for more than $80 \%$ of the observed TKE. The remainder could be attributed to measurement error, to randomness of the original data, or to unknown upwind behaviour not captured by the local fluxes. In the daytime, however, there is a consistent departure that is a strong function of the height of measurement (approximately $\propto Z^{-1 / 2}$ ). The causes of this apparent departure remain to be examined.

The results shown in Fig. 7 derive from the use of $u_{*}$ as the relevant parameter for consideration. There is little change if the analysis is alternatively based on $u_{*}^{3}$, although the depth of the daytime "well" is slightly reduced. Reliance on $u_{*}^{3}$ would be more aligned with understanding of the generation of TKE by drag forces, but it appears that the statistical methodology is largely unaffected by the precise formulation of the assumed causative properties.

\section{Turbulence Structure}

It is acknowledged that the correlation coefficients represent only part of the overall interpretation. Figure 8 offers a different approach: it shows how the average turbulence shape ratios $\sigma_{v} / \sigma_{u}(8 \mathrm{a})$ and $\sigma_{w} / \sigma_{u}(8 \mathrm{~b})$ change through the morning transition and the subsequent period. The red and blue lines indicate (as before) data from the $8.5-\mathrm{m}$ and $21.0-\mathrm{m}$ levels. In
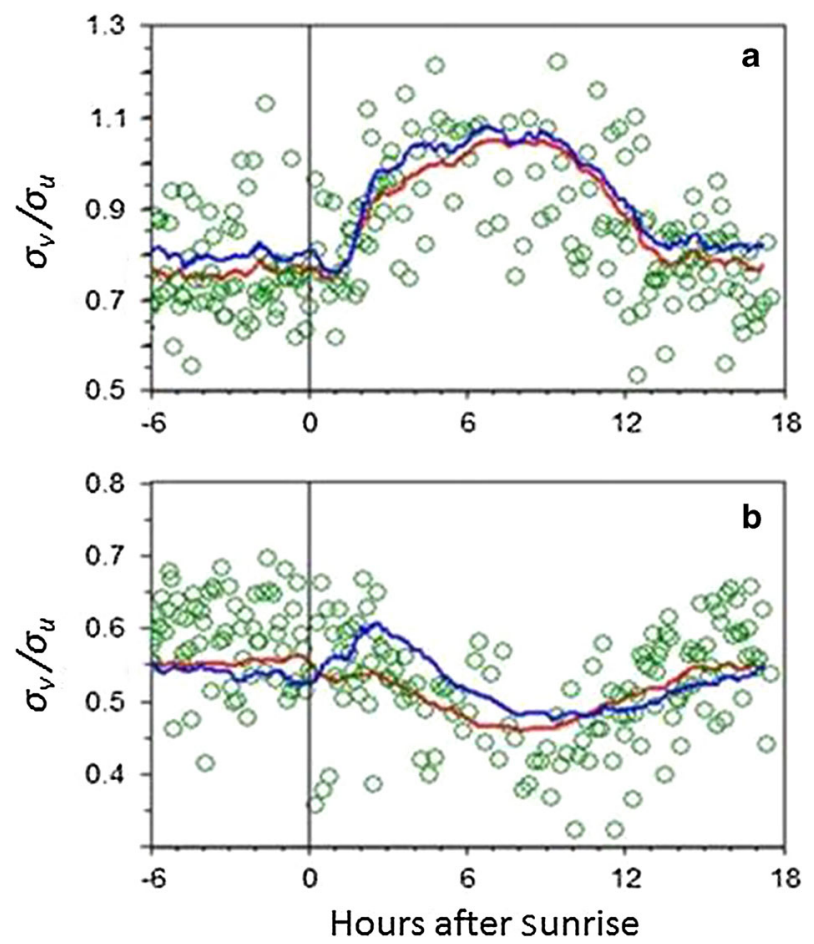

Fig. 8 The average diurnal cycles of the turbulence shape ratios $\sigma_{v} / \sigma_{u}$ (a) and $\sigma_{w} / \sigma_{u}(\mathbf{b})$. Red, green and blue refer to data from levels $8.5,14.7$, and $21.0 \mathrm{~m}$. Every fiftieth point is plotted for the $14.7-\mathrm{m}$ data, to give an indication of the scatter of the raw data 

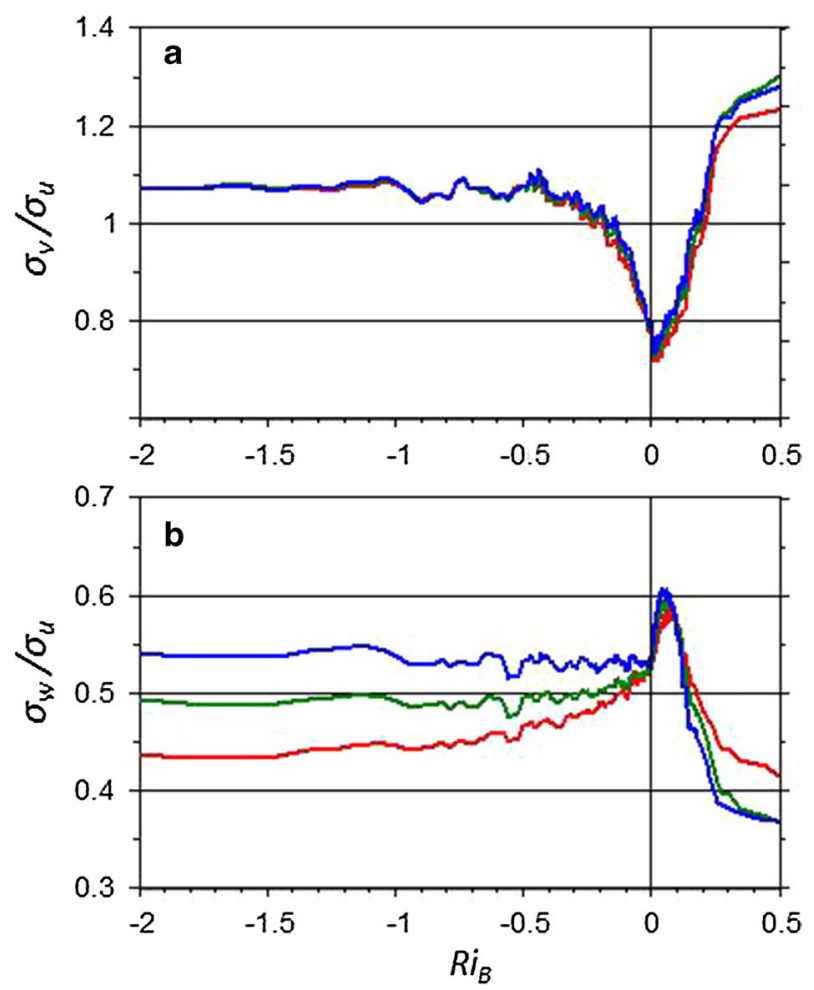

Fig. 9 The stability dependence of the turbulence shape ratios $\sigma_{v} / \sigma_{u}$ and $\sigma_{w} / \sigma_{u}$. Data for the same three heights as in Fig. 8 are shown: $8.5 \mathrm{~m}$ (red), $14.7 \mathrm{~m}$ (green), and $21.0 \mathrm{~m}$ (blue)

Fig. 8a and b, the scatter associated with the averages plotted in red and blue is exemplified using the 14.7-m data (green), for which every fiftieth point is plotted after ordering by time (hours after sunrise). The scatter is about the same for all of the data records of this kind. Note that, as before, blue and red lines are defined by the averages constructed over sequential groups of 50 data runs. The averages of the $14.7-\mathrm{m}$ data lie between those of the $8.5-\mathrm{m}$ and the 21.0-m data. These averages are based on all of the data, not just on those (2\%) that are plotted.

Figure 8a indicates that the average eddy structure before dawn is elongated longitudinally. After sunrise, $\sigma_{v} / \sigma_{u}$ starts to increase until it appears to reach a plateau at a value close to unity, followed by a slow decline as sunset approaches. The onset of a trend towards $\sigma_{v} / \sigma_{u} \approx 1$ does not start at sunrise, but is delayed by about $1 \mathrm{~h}$ with the onset of instability. In comparison, Fig. 8 b shows relatively little variation in $\sigma_{w} / \sigma_{u}$ following dawn. Figure 8 does not generate strong support for relying on the time of day as a controlling factor, and Fig. 9 indicates that stability is a better means of describing the variation of the turbulence shape functions. Data from three heights are shown, 8.5, 14.7 and $21.0 \mathrm{~m}$, as functions of $R i_{B}$. In the case of $\sigma_{v} / \sigma_{u}$ (Fig. 9a), data from all of the levels tend to overlie each other, but this is not the case for $\sigma_{w} / \sigma_{u}$ (Fig. 9b). The consistency of the unstable value of $\sigma_{v} / \sigma_{u}$ at a value exceeding unity is unexpected and unexplained. This could be either a consequence of the method of analysis (employing linear averaging of data that tend to be log-normally distributed) or a repercussion of an instrumental effect such as that reported by Kochendorfer et al. (2012). 

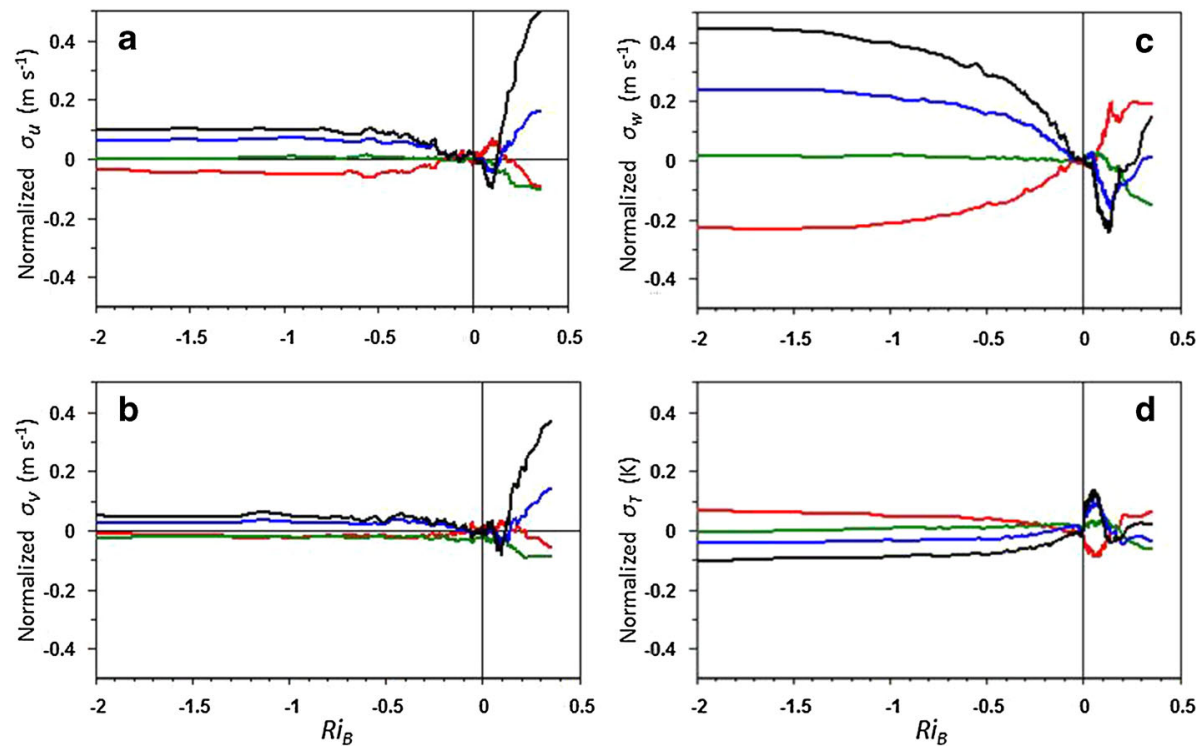

Fig. 10 The variation with stability of the three turbulent velocity components, $\sigma_{u}, \sigma_{v}$ and $\sigma_{w}$, and of the corresponding standard deviation of temperature, $\sigma_{T}$. The curves plotted represent departures from neutral, after normalization using the average value of $\sigma_{u}$ (for example) derived from observations at the five central levels of measurement. Data for four heights are shown-8.5 m (red), $14.5 \mathrm{~m}$ (green), $21.0 \mathrm{~m}$ (blue), and 27.4 $\mathrm{m}$ (black)

Consider the unstable case: given that the fluxes are expected to be constant with height within the SBL, and hence that the Obukhov length scale $L$ is constant with height, the assumption that the velocity component standard deviations when normalized by $u_{*}$ vary with $Z / L$ requires that $\sigma_{u}, \sigma_{v}$ and $\sigma_{w}$ must change with height in a consistent manner. To explore the validity of this expectation, for each 15-min data period values of $\sigma_{u}$ for the five central levels of measurement have been averaged (i.e., using the $8.5-\mathrm{m}, 10.0-\mathrm{m}, 14.7-\mathrm{m}$, 17.2-m and 21.0-m observations). Then the departures of each of the seven determinations of $\sigma_{u}$ (expanding the set to now include the 3.0-m and 27.4-m levels) have been normalized according to this average. In this way, a dimensionless and normalized profile of $\sigma_{u}$ has been derived for every 15-min "run." The averaging procedure adopted before has then been applied, with averaged normalized profiles being computed for sequential groups of fifty runs after sorting according to $R i_{B}$. An identical procedure was then used for the other velocity standard deviations, $\sigma_{v}$ and $\sigma_{w}$, and for $\sigma_{T}$. Results from six of the seven levels of measurement are plotted in Fig. 10. As before, data from the 3.0-m level are not represented because of their proximity to surface vegetation. It is clear that there is little variation of $\sigma_{u}$ with height in unstable conditions, and less variation of $\sigma_{v}$ (of questionable significance), but that there is a strong and consistent variation of $\sigma_{w}$. The widely espoused relationship with $(-Z / L)^{1 / 3}$ is supported by the observations mainly for the case of the vertical velocity. In stable conditions, all of the data records become disordered beyond $R i_{B} \approx 0.1$.

Elsewhere, it was concluded that the neutral velocity profiles indicate a zero-plane displacement $d=0.8 \pm 0.4 \mathrm{~m}$ (Hicks et al. 2014). Consideration of the dependence of $\sigma_{w}$ on height reveals that for strongly unstable conditions the highest correlation between $\sigma_{w}$ and height results if the height scale is referred to the surface itself, without allowance for a nonzero zero-plane displacement. This result is obtained regardless of whether the $3.0-\mathrm{m}$ data 
are included in the analysis. This is reminiscent of past discussions on whether zero-plane displacements need be common for all properties (q.v. Garratt 1978; Raupach et al. 1979) or can be taken as variables affected by the local situations and circumstances (Hicks et al. 1979).

\section{Discussion}

The present reliance on data obtained over 15-min averaging periods raises questions about the most desirable run length. The present choice was made to provide the finest time resolution compatible with standard micrometeorological requirements. Two tests have been made to ensure the adequacy of the 15-min sampling protocol. First, the original data record has been subjected to a three-point running mean, essentially imposing a 45-min run length on the data stream. Second, a subsequent period of data collection using 1-hr sampling took place during October and November 2011. Neither of these alternative treatments indicate a need to refine the analysis or the results obtained using the original 15-min data, as reported here, except for extremely stable or unstable cases. Further examination of these extreme cases appears warranted.

The earlier examination of the Ocotillo data from the perspective of flux-gradient relations (Hicks et al. 2014) supported a straightforward picture of the evolution of the surface boundary layer. At night, the surface cools radiatively and a vertical temperature gradient evolves in response to this and the prevailing turbulence regime. This turbulence regime results mainly from surface friction and the flow aloft. The mechanically generated turbulence and the radiatively-imposed temperature gradient determine the flux of heat. The eddy transfer at night will be affected by the restraining influence of negative buoyancy. For the case of the arid surface considered here, with sparse vegetation, immediately after sunrise surface heating generates low-level convection that erodes the near-surface stratification thus allowing the development of deep convection. (The earlier analysis indicated that this "barrier" corresponds to a value of $R i \approx-0.03$.) At all heights, vertical wind components reflect this change but will gradually relax to a more coherent field of free convection within a moving air mass. Once convection starts to diminish at the end of the day, there is a transition to the evening case of relatively constant correlation among all of the variables presently considered, but with the turbulent fluctuations becoming more disordered as stability increases.

Comparison between Figs. 2 and 3 indicates that the standard errors associated with the averages plotted are similar, regardless of whether the grouping is by $R i_{B}$ or time after sunrise (with the periods near dusk and dawn being obvious exceptions). Thus, if it is desired to reflect the variability characteristic of the plotted quantities, then it appears to be immaterial whether the depiction is based on stability or on time.

Before the convective regime emerges, Fig. 8a shows that eddies are stretched longitudinally $\left(\sigma_{v} / \sigma_{u} \approx 0.75\right)$. When the convective regime is fully developed, the shape ratio approaches unity, so that the data are then indicative of a circular pattern. Figure 9a supports the conclusion elsewhere that $\sigma_{u}$ and $\sigma_{v}$ tend to equilibrate as instability increases, and it follows that in unstable conditions the eddy structure moves gradually from elongation in the along-wind direction to a more circular structure as free convection takes over. However, this interpretation of the data is clouded by the observation that the ratio of $\sigma_{v}$ to $\sigma_{u}$ tends to a value (obvious in Fig. 9a) exceeding the expected value of 1.0. It remains unclear whether this reflects a characteristic of the atmosphere, an artifact of the anemometers used (q.v. Kochendorfer et al. 2012), or a consequence of the present methods of analysis. 
Note that "free convection" is not necessarily associated with daytime periods of calm. The derivation of the relationships describing free convection requires only that the friction velocity does not affect the convective heat transfer, regardless of the speed at which the air mass moves past an observing point. The partial correlation coefficients shown in Fig. 6 align well with this understanding, independently of whether the basis is $R i_{B}$ (Fig. 6a) or $Z / L$ (Fig. 6b). The detail evident in the behaviour for stable conditions and its apparent variability has not been explored further (since additional analysis would seem to require consideration of spatial aspects of the fluxes, for which data are lacking).

Figure 7 illustrates that local fluxes account for most (>80\%) of the local TKE for much of the diurnal cycle. However, the fraction of TKE that can be explained in terms of local fluxes decreases as daytime instability sets in; this apparent departure increases consistently with height. These results are certainly affected by randomness (scatter) and by errors (both in measurements and, possibly, in analysis). It is also possible that the use of a 15-min run time could be a contributing factor. For the present, it is sufficient to note that near noon the local fluxes appear to account for a reduced proportion of the local TKE.

The concept of an upwind area that is associated with the flux measured at some height above the surface has been explored extensively. In recent years, the issue has received attention from the perspective of the total PBL rather than the SBL alone (Wilson and Swaters 1991) and most recently as it affects the interpretation of scalar fluxes (Kljun et al. 2004). In the intervening years, there were many examinations of this issue, most of which were based on models that (as emphasized above) assumed some sort of atmospheric "well-behaved" continuity. The present analysis suggests that the association of TKE with local sensible heat and momentum fluxes is weakest at that part of the day when the sensible heat flux is the greatest.

It remains to establish the averaging times and/or areas over which the understanding of the averages permit estimation of the requisite micrometeorological quantities (e.g. the correlation coefficients) to a desired accuracy, say within $\pm 10 \%$. Examination of the scatter of data constrained within ranges of stability or time-since-sunrise indicates that this $\pm 10 \%$ target could be attained by combining data from (typically) about ten 15-min runs. This number is not constant, but varies in a manner that cannot be well addressed using the present single-location dataset. The scales of applicability are therefore of the order of hours or $10 \mathrm{~km}$ or more. Applying the legacy flux-gradient relationships over scales smaller than these without allowance for randomness would appear to be courageous, if not inappropriate.

All of these results are possibly influenced by characteristics of the Ocotillo site, which is located in an area of unusually high winds. Moreover, the site is routinely affected by a nocturnal jet at heights usually in the range of $300-500 \mathrm{~m}$. The occurrence of jets of this kind is characteristic of the entire region, and has been closely studied using data obtained elsewhere in the Southern Great Plains region (Whiteman et al. 1997). Such jets could intermittently disrupt the vertical exchange at night through bursts of turbulence propagating through the boundary layer beneath the jet (Nappo 1991; Banta 2008; Mahrt 2014).

Subsequent to the study reported here, a short period of study based on 1-h averaging was conducted. Analysis of this second dataset yields results that do not differ greatly from those presented here, except for the stability extremes. One consequence is that the 1-h data yield a depiction of the well in Fig. 7 that is slightly shallower, apparently supporting the conclusion that a dominant factor is the ability of a single tower to sample a wide field of convective activity with run-to-run consistency. 


\section{Conclusions}

The present analysis intentionally avoids relying on $Z / L$ as the sole stability index, so as to reveal variations in the characteristics of turbulence without imposing misleading correlations through the shared variable syndrome. Averaging according to the bulk Richardson number between heights of 8.5 and $21.0 \mathrm{~m}$ indicates a consistent departure of the $R_{w T}$ correlation coefficients from $R_{w u}$ in strongly stable situations, whereas this feature is obscured if averaging is by time of day. Averaging according to time of day (relative to sunrise) reveals that the erosion of the nocturnal stratification at the Ocotillo site commences immediately at sunrise, as soon as the surface is exposed to insolation, and that this convection erodes the pre-existing stratification until eventually the stratification obstructions to deep convection are destroyed. This finding emphasizes the importance of considering surface biomass in predicting the rate of change of surface temperature (and sensible heat flux) with time following sunrise.

The Ocotillo dataset provides strong evidence concerning the dominant role of randomness (or of processes beyond the local surface boundary layer); this variability cannot be attributed to sensor limitations - it is a feature of the atmosphere. All relevant legacy relationships are based on descriptions of averages, constructed by combining observations into groups according to (most often) stability. Here, it is shown that analysis according to time of day can yield much the same depiction of variability as analysis by stability.

Micrometeorological convention relates turbulence quantities to the characteristics of the local site and the prevailing heat and momentum fluxes (neglecting here, for convenience, the role of humidity). The Ocotillo data indicate that this view is most appropriate near neutral and in stable (nighttime) situations. During the daytime, the proportion of the local TKE that can be locally attributed appears to decrease consistently with the height of measurement. Insofar as applications such as dispersion rely on formulations of turbulence statistical properties, predictive models could be faced with accounting for additional turbulence contributions than are associated solely with local fluxes, to an extent that is expected to be site specific (q.v. Zilitinkevich 1995) and strongly influenced by scatter. It is currently proposed to explore this matter further, by applying the analytical methods employed here to other datasets.

Consideration of the variations in turbulence statistics following sunrise leads to several observations that appear to deviate from conventional expectations. First, the conclusions drawn can be influenced by the way in which the initial data are organized. As an obvious example, averaging according to stability imposes a merging of datasets from both the morning and evening transitions, and the present analysis shows that these can differ substantially. Second, the present analysis confirms that large uncertainties arise whenever relationships among averages are used to describe specific SBL situations. The uncertainties that arise appear to be similar, regardless of the basis for initial estimation-stability or time of day.

The present data indicate that many 15 -min runs would be necessary to reduce the resulting uncertainty to an acceptable level (say, $\pm 10 \%$ ) if considerations are based on relationships among averages alone. The implicit conclusion is that the size of the grid over which averages should be constructed for purposes of comparison with numerical model predictions (or conversely for the prediction of near-surface meteorological conditions) must likely be $>10 \mathrm{~km}$. Smaller than that, randomness will present a challenge for all deterministic simulations. It remains to be seen whether such conclusions have direct relevance to current numerical models, which are in the process of DECREASING grid sizes, and hence probably of INCREASING levels of uncertainty relevant to any selected location within the surface boundary layer. 
Acknowledgments The data used here were collected as a part of an investigation of the planetary boundary layer in support of the wind energy program of Duke Power, Inc., through a formal agreement with the Air Resources Laboratory of NOAA. An unknown reviewer provided some especially useful insight that helped clarify the presentation.

Open Access This article is distributed under the terms of the Creative Commons Attribution License which permits any use, distribution, and reproduction in any medium, provided the original author(s) and the source are credited.

\section{References}

Banta RM (2008) Stable-boundary-layer regimes from the perspective of the low-level jet. Acta Geophys $56: 58-87$

de Heus P (2012) R squared effect-size measures and overlap between direct and indirect effect in mediation analysis. Behav Res 44:213-221

Garratt JR (1978) Transfer characteristics for a heterogeneous surface of large aerodynamic roughness. Q J R Meteorol Soc 104:491-502

Hicks BB, Hess GD, Wesely ML (1979) Analysis of flux-profile relationships above tall vegetation-an alternative view. Q J R Meteorol Soc 105:1074-1077

Hicks BB, Pendergrass WR III, Keener RN Jr, Leyton SM, Vogel CA (2014) On the micrometeorology of the Southern Great Plains 1: legacy relationships revisited. Boundary-Layer Meteorol 151:389-405

Kljun N, Calanca P, Rotach MW, Schmid HP (2004) A simple parameterization for flux footprint predictions. Boundary-Layer Meteorol 112:503-523

Kochendorfer J, Meyers TP, Frank J, Massman WJ, Heuer MW (2012) How well can we measure the vertical wind speed? Implications for fluxes of energy and mass. Boundary-Layer Meteorol 145:383-398

Mahrt L (2014) Stably stratified atmospheric boundary layers. Ann Rev Fluid Mech 46:23-45

Nappo CJ (1991) Sporadic breakdowns of stability in the PBL over simple and complex terrain. BoundaryLayer Meteorol 54:69-87

Priestley CHB (1955) Free and forced convection in the atmosphere near the ground. Q J R Meteorol Soc 81:139-143

Raupach MR, Stewart JB, Thom AS (1979) Comments on the paper "Analysis of flux-profile relationships above tall vegetation-an alternative view” by Hicks BB, Hess GD, Wesely ML (Q. J., 105, 1074-1077). Q J R Meteorol Soc 105:1077-1078

Whiteman CD, Bian X, Zhong S (1997) Low-level jet climatology from enhanced rawinsonde observations at a site in the Southern Great Plains. J Appl Meteorol 36:1363-1376

Wilson JD, Swaters GE (1991) The source area influencing a measurement in the planetary boundary-layer: the footprint and the distribution of contact distance. Boundary-Layer Meteorol 55:25-46

Zilitinkevich S (1995) Non-local turbulent transport: pollution dispersion aspects of coherent structure of convective flows. Trans Ecol Environ 6:53-60 